This item is the archived peer-reviewed author-version of:

The vicious cycle of arterial stiffness and arterial media calcification

\title{
Reference:
}

Van den Bergh Geoffrey, Opdebeeck Britt, d' Haese Patrick C., Verhulst Anja.- The vicious cycle of arterial stiffness and arterial media calcification Trends in molecular medicine - ISSN 1471-4914 - Oxford, Elsevier sci Itd, 25:12(2019), p. 1133-1146

Full text (Publisher's DOI): https://doi.org/10.1016/J.MOLMED.2019.08.006 


\section{The Vicious Cycle of Arterial Stiffness and Arterial Media Calcification}

Geoffrey Van den Bergh ${ }^{1} \mathrm{MSc}^{*}$, Britt Opdebeeck ${ }^{1} \mathrm{MSc}^{*}$, Patrick C D'Haese ${ }^{1} \mathrm{PhD}$, Anja Verhulst ${ }^{1} \mathrm{PhD}$.

*Equally contributed to

${ }^{1}$ Laboratory of Pathophysiology, Department of Biomedical Sciences, University of Antwerp, Belgium

\section{Corresponding author and offprint request:}

Patrick C. D'Haese, PhD

Laboratory of Pathophysiology, University of Antwerp, Campus Drie Eiken

Universiteitsplein 1

B-2610 Wilrijk Tel: +32-3 2652599 Fax: +32-3 2652592 patrick.dhaese@uantwerpen.be

Key words: Arterial stiffness - arterial media calcification - pulse wave velocity phenotypic transition - endothelial dysfunction - mechanosensing

\section{Abstract}

Arterial media calcification and arterial stiffness are independent predictors of cardiovascular mortality. Both processes reinforce one another, creating a vicious cycle in which transdifferentiation of endothelial cells and vascular smooth muscle cells play a central role. Physiological functioning of vascular smooth muscle cells in the arterial medial layer greatly depends on normal endothelial cell behavior.

Endothelial or intimal layer cells are the primary sensors of pathological triggers circulating in the blood during for example ageing or inflammation, and often can be seen as initiators of this vicious cycle. As such, the search for treatment of arterial media calcification, which until now mainly concentrated at the level of the vascular smooth cell may need to be expanded to intimal layer targets. 


\section{From Physiology to Pathology, Arterial Stiffness in a Nutshell}

A large and rapidly growing number of studies are now investigating the non-invasive measurement of arterial stiffness in vivo and how these measurements are related to cardiovascular risk and disease prognosis. Aortic stiffness, measured through carotid-femoral pulse wave velocity (PWV, see Glossary) assessment, has emerged as an important independent predictor of cardiovascular events and overall mortality [1, 2].

A proximal to distal stiffness gradient is present in the arterial tree (box 1) and increase in arterial stiffness is associated with an increase in carotid femoral PWV. Earlier arrival of pulse wave reflections, during systole, augments systolic pressure and reduces diastolic pressure. Principal consequences are superimposed leftventricular overload and negatively affected coronary perfusion pressure which naturally occurs during diastolic filling. The mismatch between oxygen demand and reduced oxygen delivery may result in myocardial ischemia. In addition, arterial stiffening usually involves the large proximal aorta. This results in a decline or even a reverse of the normally present stiffness gradient. Consequently, the propagating wave is able to penetrate more distally into the arterial system, this time not partly withheld by reflection from the resistance vessels $[3,4]$. Ultimately, these events lead to an increased transmission of pulsatile energy towards strongly perfused, low resistance organs, such as the kidneys and brain, with potentially damaging consequences $[5,6]$.

Traditionally, mechanisms of central arterial stiffening include passive stimuli, linked to elementary material properties, i.e. an increased mechanical stress due to distending pressure that leads to structural disorganization and fragmentation of elastic fibers, either by matrix metalloproteinases (MMPs) or material fatigue [7]. 
The search, however, for cell-mediated (active) mechanisms underlying progressive arterial stiffening and for interventions to halt or reverse this process has gained much attention. Cellular mechanisms inducing arterial stiffness i.e. endothelial dysfunction and vascular smooth muscle cell (VSMC) transdifferentiation will be focused on in this review, in particular their role in arterial media calcification (AMC) will be discussed. Furthermore, mechano-sensing of endothelial cells (ECs) and VSMCs, as well as mesenchymal stem-like cell transition of ECs, might provide novel insights in the molecular mechanisms underpinning AMC and central arterial stiffening. These processes might be the driving force in the vicious circle of arterial stiffness inducing $\mathrm{AMC}$ and vice versa. Figure $1 \mathrm{~A}$ summarizes the focus of this review.

\section{Endothelial Cells Influence Vessel Tone}

The internal surface of the vasculature consists of a single cell layer of heterogeneous ECs (intimal layer). The strategic positioning of the endothelium allows it to sense hemodynamic changes and to adequately respond to these changes. As such, the endothelium is playing a crucial role in maintaining vascular homeostasis, maintaining a balance between endothelium-derived relaxing and contracting factors [8]. Adequate cooperation between functional ECs and VSMCs of the medial layer is required to fulfil this task. Disturbances in this balance predispose the vessel to increased vessel tone, pro-inflammatory state, oxidative stress and impaired coagulation [9]. Regulation of vessel tone by ECs and its relation to arterial stiffness will be discussed first in this review and is summarized in Figure 2. Physiological vessel tone regulation is facilitated by mechanical stimuli and vasoactive molecules (box 2). 
Impaired Vasorelaxation: Culprit of Arterial Stiffness

Local net bioactive nitric oxide (NO) increases large artery distensibility in vivo as was concluded from a significant (6\%) higher PWV in the absence of NO [10]. Also, muscular artery distensibility is modulated by local NO action [11]. In healthy male volunteers, hemodynamic measurements during infusion of vasopressor drugs for 30 minutes, resulted in inhibition of the basal NO production and a significant increase in central artery stiffness (higher carotid-femoral PWV) in comparison to saline infused healthy volunteers. This effect however, could largely be explained by a concomitant increase in blood pressure (which is also regulated by muscular arteries) rather than being fully NO-mediated [12]. Controversial findings like this could partly be explained by the heterogeneity in the arterial tree: elastic arteries are more subject to arterial stiffness (with increasing age) compared to muscular arteries [13].

Endothelial-derived endothelin-1 (ET-1) promotes vasoconstriction through binding to $\mathrm{ET}_{\mathrm{A}}$-receptors on VSMCs, thereby directly regulating central artery distensibility, resulting in an elevation of the aortic PWV [14]. Chronic administration of an $\mathrm{ET}_{\mathrm{A}}$ receptor antagonist to rats reverses hypertension and recovers endothelial function and flow dynamics in smaller peripheral vessels [15]. Angiotensin II receptor antagonists, on the other hand, induce a decrease in blood pressure independent of aortic stiffness and wave reflections, as was observed in humans [16].

These findings highlight the hemodynamic coupling between central aortic stiffening and peripheral arterial vessel flow dynamics. Regulation of arterial compliance clearly relies on the vessel type and must be a summation of the complex interaction between large elastic and smaller muscular arteries of the periphery. 
In normal physiological conditions, constant distending pressure on proximal arteries causes cyclic stretch to be larger in elastic arteries compared to smaller muscular arteries. Since basal NO production is highly dependent on the stimulation of cyclic stretch, elastic arteries display higher basal production and release of NO compared to muscular arteries $[17,18]$. Relaxation of VSMCs in muscular arteries is mainly featured by the process of endothelium-dependent hyperpolarization (EDH), as described below.

In pathophysiological conditions, when cyclic stress is elevated, altered wall mechanics promote large artery stiffening and organ related damage [19].

\section{Endothelium Induced Hyperpolarization of Vascular Smooth Muscle Cells}

Thus far, local vasoactive molecules, of which NO and ET-1 are the most important, influence proximal artery distensibility. The endothelium also mediates relaxation of VSMCs via EDH [20]. Endothelial derived hyperpolarizing factors (EDHFs) involved in this process are only partially understood. There is no such thing as a single universal EDHF. Over the years, many discoveries have led to a basic concept of EDH: calcium influx through transient receptor potential vanilloid 4 (TRPV4) or store operated calcium (SOC) channels facilitates an elevation of endothelial intracellular calcium, in response to acetylcholine (ACh) or shear stress [21-23]. Increased

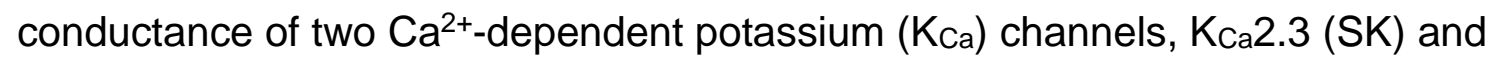
Kca3.1 (IK) leads to a larger potassium-pool in the interstitial space. This, in turn activates inwardly rectifying potassium channels, large-conductance $\mathrm{K}_{\mathrm{ca}}$ channels and $\mathrm{Na}^{+} / \mathrm{K}^{+}$-ATPases in VSMCs, thereby facilitating relaxation through hyperpolarization [24]. Simultaneously, this hyperpolarizing current can spread to subjacent VSMCs through myoendothelial gap junctions (MEGJs), and 
subsequently spreads through adjacent VSMCs through homocellular gap junctions [25].

NO-dependency diminishes progressively along the vascular tree. In contrast, the role of EDH may be of particular importance in muscular conduit arteries and smaller resistance arteries, regulating peripheral resistance and blood pressure [26].

Likewise, the number of MEGJs and associated gap junctions is twice as high (significantly higher) in distal arteries compared to proximal arteries [27], highlighting the close relationship between EDH-dependent relaxation and gap junctions. Therefore, most research regarding EDH has been conducted on muscular arteries.

EDH-mediated dilation is virtually absent in mice deficient for both SK and IK channels. Moreover, these mice showed significantly elevated arterial blood pressure levels and mild left ventricular hypertrophy [28]. Furthermore, in mice, a polymorphism in endothelial connexin40, a gap junction protein facilitating the hyperpolarizing current, increases arterial stiffness [29]. Genetic animal studies thus confirmed the important contribution of EDH to arterial distensibility.

Lastly, vasoactive molecules such as ACh trigger arachidonic acid metabolism in ECs by cytochrome P450 (CYP450), resulting in the generation of epoxyeicosatrienoic acids (EETs). These latter effector molecules have been shown to induce VSMC relaxation by contributing to the EDH response, stimulating channel conductance on both ECs and VSMCs [30, 31]. Another metabolite of arachidonic acid metabolism, 20-hydroxyeicosatraenoic acid (20-HETE) has opposite effects causing vasoconstriction through blocking large conductance Kca channels and stimulating calcium influx in VSMCs [31]. Recently, in a rat model of metabolic syndrome, production of 20-HETE increased $\sim 7$ fold compared to control rats in large conduit arteries (carotid artery and aorta), contributing to arterial stiffness [32]. 
151 Increased levels of EETs act as a partial compensatory mechanism to sustain

152

153

154

endothelium-dependent vasodilation in muscular arteries of hypertensive patients, in particular when bioavailable NO is decreased because of oxidative stress [33]. More recently, synergic contribution of NO and EETs has been implicated in aortic wall viscosity, i.e. cardiac energy dissipation along the arterial tree [34]. These findings highlight that EETs support vessel tone regulation and are potentially protective against arterial stiffening.

EDH-dependent relaxation, with a regulating role for CYP450, in muscular conduit arteries and smaller resistance arteries may modulate central arterial stiffness.

\section{Arterial Media Calcifications: More Than a Vascular Smooth Muscle Cell}

\section{Regulated Process}

Vascular calcification is an active cell-regulated process depending on (i) the production of osteoblast-like cells facilitating the deposition of calcium-phosphate crystals into an organized extracellular matrix and (ii) an imbalance between circulating calcification stimulators and inhibitors. This process can occur at two anatomical sites being the vessel intimal layer during atherosclerosis or the vessel medial layer in ageing, chronic kidney disease (CKD) and diabetes each of them having their typical clinical complications [35]. In this review, we will focus on the mineralization of the medial layer, so-called AMC. Arterial stiffness and AMC are independent predictors of cardiovascular morbidity and mortality $[3,36]$ and many clinical studies have shown a strong correlation between both [37-39]. Furthermore, AMC and arterial stiffness both are hallmarks of ageing and notably, within the patient populations of CKD and diabetes, both AMC and arterial stiffness may 
already be present at young age $[38,39]$. As such, besides age, risk factors of arterial stiffness and AMC can directly be attributable to either diabetes or CKD: hyperglycemia, obesity, hypertension, hyperlipidemia, insulin resistance, inflammation, oxidative stress, uremic toxins, hyperphophatemia and low bone mineral density [40].

Endothelial Contribution to Arterial Media Calcification - Impaired NO Regulation

Endothelial dysfunction, and in particular impaired NO regulation, might play a role in the pathophysiology of $A M C$. In klotho deficient mice, vitamin $\mathrm{D}_{3}$ overloaded mice and $\mathbf{5} / \mathbf{6}^{\text {th }}$ nephrectomized mice, which all are models for AMC, treatment with homoarginine increased calcification and osteochondrogenic transdifferentiation of VSMCs, probably by impaired NO formation [41]. In vitro, NO inhibits murine VSMCs calcification and osteochondrogenic transdifferentiation via inhibition of TGF $\beta$-induced phosphorylation of SMAD2/3 [42] whilst metformin halts AMC via restoration of NO bioavailability (via the AMPK-eNOS-NO pathway) in rats $[43,44]$. These findings suggest a role for NO in calcification of the medial layer of the arterial wall. Consequently, a reduction in bioavailable NO may be more impactful than solely affecting vessel tone by influencing active (cellular) stiffness components.

Endothelial Contribution to Arterial Media Calcification - Endothelium as a Source of Multipotent Cells

ECs possess the ability to undergo endothelial to mesenchymal transition (EndMT), a subtype of epithelial to mesenchymal transition (EMT) which involves ECs (Figure 3). EndMT has gained increasing attention in cancer research [45, 46]. EndMT results in the generation of mesenchymal stem-like cells that can differentiate further into multiple cell lineages: fibroblasts/myofibroblasts, osteoblasts/osteocytes, 
chondrocytes and/or adipocytes [47]. The EC obtains an elongated phenotype losing its cell-cell connections, enabling migration through the basal lamina, which is degraded by MMPs such as MMP-2 and MMP-9 and replaced by new extracellular matrix molecules like type I collagen, type III collagen and fibronectin [47].

During EndMT, the expression of endothelial markers such as vascular endothelial (VE)-cadherin, CD31, von Willebrand Factor (vWF), Tie1 and Tie2 is significantly reduced [48]. Furthermore, the transforming cells acquire mesenchymal stem cell (MSC) markers such as alpha smooth muscle actin ( $\alpha-S M A)$, smooth muscle protein 22 alpha (SM22a), fibroblast-specific protein 1 (FSP-1) and others [49]. EndMT can be promoted by a myriad of factors, of which the TGF- $\beta$ /bone morphogenetic protein (BMP) family has been most widely described. Especially the TGF- $\beta 2$ isoform is a strong promotor of EndMT [50]. Additionally, BMP2 and BMP4 have been shown to promote EndMT. Both ligands primarily signal through the activin-like kinase 2 (ALK2) and ALK5 receptors, resulting in downstream activation of SMAD1/5/8 and SMAD2/3 complexes and nuclear shuttling of these complexes by SMAD4 [47]. Complexation with transcription factors such as Snail, Slug and Twist results in EndMT activation and subsequent degenerative remodeling of the arterial wall [51]. BMP7 and vascular endothelial growth factor (VEGF) inhibit EndMT by binding to ALK2 and VEGF-receptor 2, respectively [52, 53]. Fibroblast growth factor-2 (FGF-2) is another inhibitor of EndMT through down-regulation of TGF- $\beta$ signaling [54]. Interestingly, in mineralizing VSMCs, FGF-2 is upregulated as a feedback mechanism to prevent more extensive mineralization [55].

Although TGF- $\beta$ /BMP signaling is considered the main regulator of EndMT, alternative signaling pathways might cooperate. Notch signaling and wnt/ß-catenin signaling are involved in cardiac cushion differentiation during embryonic 
endocardial development, a process which is believed to involve EndMT [56, 57]. Various signaling pathways are able to induce EndMT. Ultimately, which pathways specifically determine the fate of the EC remains elusive. Yet, the complex regulatory system accompanying EndMT highly suggests that mesenchymal transition might play an important role in normal physiology and disease. Moreover, it is highly suggestive that originally embryonic pathways, such as EndMT, may become reactivated in pathological conditions when sufficient stimuli are present.

Insights from the extremely rare, autosomal dominant disorder, fibrodysplasia ossificans progressiva (FOP) shed light on the contribution of EndMT to ectopic calcification (including AMC). Constitutively active ALK2, in the absence of its ligands, predisposes fully differentiated EC to transition and acquire mesenchymallike properties, enabling them to differentiate further into osteoblast-like cells in a step wise manner [58, 59]. Furthermore, in mice lacking matrix gla protein (MGP), an endogenous BMP inhibitor and potent inhibitor of ectopic calcification (including AMC), the endothelium may act as a source of mesenchymal stem-like cells contributing to the development of AMC [60]. Moreover, enhanced BMP signaling, for example in diabetes [61], promotes EndMT and therewith associated calcifications in a Sox2 (sex determining region Y-box 2) dependent manner [62].

Initiation of EndMT has also been linked to failing vessel tone regulation of ECs. The above mentioned inhibitory actions of NO on ET-1 (and the lack thereof in disease) are relevant in this context. Indeed, ET-1 potentiates the pro-fibrotic effect of TGF- $\beta 1$ induced EndMT [63]. Moreover, chronic NO synthase (NOS) inhibition in the presence of an endogenous inhibitor triggers EndMT in vivo and in vitro [64]. Finally, calcium conductance through TRPV4, which is crucial in the initiation of EC-induced $\mathrm{EDH}$, has been shown to play a role in TGF- $\beta$ induced EMT of keratinocytes [65]. 
Functional alterations in response to vasoactive substances thus might have implications on a more complex level, contributing to EndMT regulation.

EndMT also contributes to the pathobiology of aortic valve stenosis. The constant movement of the valves exposes the leaflets to cyclic mechanical forces. Valvular ECs are directly exposed to these stresses just like EC lining the vasculature. These stimuli, possibly via an altered NO/ET-1 balance, are sufficient to induce a healthy valvular EC to undergo TGF- $\beta$ induced EndMT in 3D in vitro models [66-68]. In addition to embryonic heart development, wnt/ $\beta$-catenin signaling plays a role in endMT of valvular ECs [68]. With central artery stiffening, cyclic stress concomitantly increases. Therefore, it is not unthinkable that the less desirable process of EndMT becomes more prevalent.

\section{Vascular Smooth Muscle Cells Contribution to Arterial Media Calcification}

VSMCs can undergo a phenotypic modulation from a contractile phenotype to modulate local blood pressure, into a synthetic phenotype to repair the arterial wall after injury. However, in the presence of certain pathophysiological triggers (i.e. mineral imbalance, uremic toxins, inflammation, oxidative stress) [69], VSMCs transdifferentiate into osteochondrogenic cells, which goes along with the secretion of calcifying exosomes (matrix vesicles) or apoptotic bodies, that stimulate the deposition of calcium-phosphate crystals in the arterial wall [70]. A recent study reports that oxidative stress and/or DNA damage induce poly(ADP-ribose) production in VSMCs and that poly(ADP-ribose), delivered to the extracellular matrix (ECM) via both apoptotic bodies and in calcifying exosomes, is essential to the physicochemical process of ECM calcification [71]. Furthermore, calcifying exosomes cause propagation of the mineralization process as these calcifying exosomes are 
endocytosed by recipient VSMC. Endocytosis of calcifying exosomes in its turn leads

to activation of MAPK and NAPDH oxidase signaling and release of calcium from the endoplasmic reticulum, ultimately contributing to an osteochondrogenic phenotypic switch of the recipient VSMCs [72]. Ultimately, structural wall rigidity through mineralization of the ECM leads to a reduction in artery compliance. On the other hand, arterial stiffness can also promote the development of AMC.

\section{The Vicious Cycle Between Arterial Media Calcification and Arterial Stiffness} Mechanosensing by Vascular Smooth Muscle Cells

When performing an in-depth synthesis of the mechanistic relationship between AMC and arterial stiffness, a vicious cycle can be observed as shown in Figure 4. VSMCs are key players in this vicious cycle. Crosslinking of adjacent collagen fibers, fragmentation of elastin fibers and overproduction of ECM products modulate the ECM of a stiffening artery. This arterial wall remodeling creates a pro-calcifying environment as elastic fragments and collagen type 1 fibers form an excellent nidus for hydroxyapatite crystal nucleation [73]. Also, the remodeling enzymes or MMPs have been implicated in AMC since their activation induces VSMC phenotypic switching $[74,75]$. Additionally, alterations in the ECM composition induce a degree of matrix stiffness which could be sensed by the VSMC, a process called mechanosensing. Further investigation of mechano-signaling through changes in wall compliance may provide additional insights in the pathophysiology of arterial stiffness.

A recent in vitro study has shown that matrix stiffness facilitates epigenetic regulation of the VSMC phenotype by down-regulation of DNA methyltransferase 1 (DNMT1) 
expression. DNMT-1 regulates the expression of critical genes maintaining the contractile phenotype of VSMCs by catalyzing the methylation of DNA [76]. In vitro matrix stiffening was mimicked with polyacrylamide gels, inducing a decrease of DNMT1 expression which in turn resulted in a contractile to synthetic phenotypic transition of VSMC as well as upregulation of the osteoblast specific proteins BMP2 and Runt-related transcription factor 2 (Runx2) [76]. Subsequently, selective overexpression of transcription factor Runx2 in mouse VSMCs resulted in collagen I accumulation in the medial layer of the aorta as well as increased matrix stiffness and arterial blood pressure [77]. Taken together, matrix stiffness-dependent upregulation of Runx2 might reinforce the development of arterial stiffness by further deterioration of the ECM as well as promote the development of AMC by inducing the transdifferentiation of VSMCs into a bone-like phenotype.

Furthermore, the mechanical stimuli sensors Yes-associated protein (YAP) and its highly related transcriptional co-activator with PDZ-binding motif (TAZ) have recently been reported as important players in the phenotypic switching of VSMCs [78]. When the cell senses matrix stiffness and cell spreading via increased cytoskeletal tension and stress fibers, the $\beta 1$-integrin-FAK-Src-PI3K-PDK1 pathway will be activated which inhibits the large tumor suppressor kinase 1/2 (LATS1/2) activity and prevents the phosphorylation of YAP/TAZ $[79,80]$. This will facilitate the nuclear translocation and transcriptional activation of YAP/TAZ target genes by interaction with TEA domain containing family transcriptional factors. Additionally, a recent study has shown that stiffening of a substrate can be sensed by the cell nucleus through the formation of focal adhesion and stress fibers that interact with the Linker of the Nucleoskeleton and Cytoskeleton (LINC) complex [81]. This leads to flattening of the nucleus and thus stretches and curves nuclear pores leading to increased YAP 
import [81]. Based on these findings, it is likely that molecules other than YAP pass through the nuclear pores during arterial stiffness and make the VSMCs more susceptible to calcification. This might be an interesting field to explore. Nuclear translocation of YAP influences transcriptional factors that are important for bone homeostasis including Runx2, PPARy and $\beta$-catenin [82]. The link between YAP/TAZ and a pro-calcifying cell phenotype is further strengthened by a study in skeletal stem cells [83]. In this cell-type YAP/TAZ collaborates with Snail and Slug, important inducers of EndMT in ECs, to activate downstream transcription of Runx2 [83]. Therefore, it might be that arterial stiffness induces an increased translocation of YAP/TAZ into the nucleus of VSMCs, which in turn could promote the upregulation of osteochondrogenic marker genes.

\section{The Endothelium, a Helping Hand in Mechano-Sensing}

Valvular ECs are able to sense matrix stiffness since EndMT of valvular ECs preferentially takes place in ECs cultured on stiff silicone substrates compared to soft ones [84]. As previously mentioned, ECs communicate with VSMCs, which might help VSMCs to sense arterial stiffness. Indeed, Rho GTPases are known to be involved in actomyosin cytoskeletal dynamics and in mechanotransduction in ECs. Matrix stiffness-dependent upregulation of Rho kinases in ECs induces endothelial permeability and a decrease of NO production [85]. An in vitro study by Peng et al. showed that ECs grown to confluence in either compliant (similar to normal carotid artery) or stiff ( $85 \%$ lower distensibility) culture tubes, exhibited reduced endothelial Akt-dependent endothelial NOS (eNOS) phosphorylation, with increasing wall stiffness [86]. Akt dependent phosphorylation of eNOS is important for vessel tone 
regulation $[87,88]$ and with regard to the study of Peng et al. decreased eNOS activity.

Reduced NO bioavailibility stimulates the VSMCs to adapt to a hypercontractile phenotype with the upregulation of genes for RhoA and Rho-associated protein kinases (ROCK1 and ROCK2) [89]. In myofibroblasts and neural crest stem-like cells, activation of the Rho/ROCK pathway respectively stimulates STAT (signal transducer and activator of transcription)3 phosphorylation [90] and YAP1 expression [91], two effectors that potentially influence VSMC behavior by (i) YAP-induced upregulation of osteoblast marker genes [82] and (ii) activation and phosphorylation of STAT3 increased Runx2 expression and calcium deposition in VSMC cell cultures [92]. Interestingly, recent studies have shown that poly(ADP-ribose) polymerase (PARP) enzyme inhibitors prevent ECM calcification by inhibiting STAT3 activation and minocycline, a PARP inhibitor, reduced AMC by more than $50 \%$ in rats $[71,92]$, thereby identifying PARP inhibitors as a novel therapeutic target to treat AMC.

During oxidative stress, observed in both AMC and arterial stiffness, NO reacts with superoxide to form a potent reactive oxygen species peroxynitrite (ONOO-) [93]. In mice, peroxynitrite mediated activation of YAP resulted in ECM remodeling by inducing collagen and fibronectin formation as well as elastin fragmentation and therewith associated with arterial stiffness [94] and potentially with AMC. Metformin reduces peroxynitrite levels in obese Zucker rats, a mechanism that may contribute to its AMC reducing effect in animal models of AMC $[43,44]$. Taken together, these results emphasize that inadequate NO production (either or not following mechanosensing of a stiffened ECM) is critical during the development of arterial stiffness and AMC as it stimulates EndMT, hypercontractility of VSMCs and production of peroxynitrite. 
Until now, the exact mechanisms by which ECs and VSMCs sense the matrix stiffness are not fully known. Identification of specific therapeutic targets in the molecular signaling pathways underlying this ECM stiffness sensing is needed as it might prevent further progression of both arterial stiffness and AMC.

\section{Concluding Remarks}

Arterial stiffness and AMC are two life-threatening diseases, implicated in cardiovascular mortality (i.e. myocardial ischemia, left ventricular hypertrophy) and end-organ failure. A big challenge presents itself for future research in this field as no effective therapies for arterial stiffness and AMC are currently available (see clinician's corner). Mineralization of the extracellular matrix induces artery wall rigidity and thus arterial stiffness, whilst the reverse is also true; i.e. arterial stiffness promoting the development of $\mathrm{AMC}$, thus creating a vicious cycle. For this reason, novel therapies against arterial stiffness could also have a beneficial effect against AMC. Functional readouts such as endothelial dysfunction, PWV and VSMC tonus, act as correlates of arterial stiffness and will be indispensable to discover the relevant disease signature(s) for both arterial stiffness and AMC. On a same note, combining physical measurements of arterial stiffness with proteomics and large database searches utilizing bioinformatics will most definitely guide us in the right direction.

As extensively described, cellular mechanisms involving ECs and VSMCs actively contribute to arterial stiffening and AMC (Figure 1B). Mechano-signaling, through changes in wall compliance, plays an important role as it influences the phenotypic transition of ECs and VSMCs. For decades, researchers working in the field of AMC focused mainly on the transdifferentiation of VSMCs into osteochondrogenic cells, and to a great extent neglected the phenotypic transition of ECs (EndMT), which 
given recent insights, might be more important than previously thought (see

as ECs are the first in line to respond to systemic stimuli and thus transmit signals to

VSMCs and activate important molecular pathways involved in both arterial stiffness

and AMC. Clarifying the exact mechanisms by which ECs and VSMCs sense matrix

stiffness is imperative to discover specific therapeutic targets to halt the progression

of arterial stiffness and AMC.

\section{Acknowledgements and funding}

G.V.d.B. and B.O. are fellows of the Fund of Scientific Research-Flanders (FWO).

\section{References}

1. Laurent, S. et al. (2001) Aortic stiffness is an independent predictor of all-cause and cardiovascular mortality in hypertensive patients. Hypertension 37 (5), 1236-41.

2. Meaume, S. et al. (2001) Aortic pulse wave velocity predicts cardiovascular mortality in subjects $>70$ years of age. Arterioscler Thromb Vasc Biol 21 (12), 2046-50.

3. Ben-Shlomo, Y. et al. (2014) Aortic pulse wave velocity improves cardiovascular event prediction: an individual participant meta-analysis of prospective observational data from 17,635 subjects. J Am Coll Cardiol 63 (7), 636-646.

4. Safar, M.E. et al. (2003) Current perspectives on arterial stiffness and pulse pressure in hypertension and cardiovascular diseases. Circulation 107 (22), 2864-9.

5. Hashimoto, J. and Ito, S. (2011) Central pulse pressure and aortic stiffness determine renal hemodynamics: pathophysiological implication for microalbuminuria in hypertension. Hypertension $58(5), 839-46$.

6. O'Rourke, M.F. and Safar, M.E. (2005) Relationship between aortic stiffening and microvascular disease in brain and kidney: cause and logic of therapy. Hypertension 46 (1), 200-4.

7. Zieman, S.J. et al. (2005) Mechanisms, pathophysiology, and therapy of arterial stiffness. Arterioscler Thromb Vasc Biol 25 (5), 932-43.

8. Sandoo, A. et al. (2010) The endothelium and its role in regulating vascular tone. Open Cardiovasc Med J 4, 302-12.

9. Michiels, C. (2003) Endothelial cell functions. J Cell Physiol 196 (3), 430-43.

10. Wilkinson, I.B. et al. (2002) Nitric oxide regulates local arterial distensibility in vivo. Circulation 105 (2), 213-7.

11. Schmitt, M. et al. (2005) Basal NO locally modulates human iliac artery function in vivo. Hypertension 46 (1), 227-31.

12. Stewart, A.D. et al. (2003) Effects of inhibition of basal nitric oxide synthesis on carotid-femoral pulse wave velocity and augmentation index in humans. Hypertension 42 (5), 915-8. 
13. Avolio, A.P. et al. (1983) Effects of aging on changing arterial compliance and left ventricular load in a northern Chinese urban community. Circulation 68 (1), 50-8.

14. McEniery, C.M. et al. (2003) Endothelin-1 regulates arterial pulse wave velocity in vivo. J Am Coll Cardiol 42 (11), 1975-81.

15. Guo, X. et al. (2018) Chronic ETA antagonist reverses hypertension and impairment of structure and function of peripheral small arteries in aortic stiffening. Sci Rep 8 (1), 3076.

16. Mahmud, A. and Feely, J. (2002) Effect of angiotensin ii receptor blockade on arterial stiffness: beyond blood pressure reduction. Am J Hypertens 15 (12), 1092-5.

17. Leloup, A. et al. (2017) Cyclic Stretch Alters Vascular Reactivity of Mouse Aortic Segments. Front Physiol 8, 858.

18. Leloup, A.J. et al. (2015) Elastic and Muscular Arteries Differ in Structure, Basal NO Production and Voltage-Gated $\mathrm{Ca}(2+)$-Channels. Front Physiol 6, 375.

19. Thorin-Trescases, N. and Thorin, E. (2016) Lifelong Cyclic Mechanical Strain Promotes Large Elastic Artery Stiffening: Increased Pulse Pressure and Old Age-Related Organ Failure. Can J Cardiol 32 (5), 624-33.

20. Feletou, M. and Vanhoutte, P.M. (2006) Endothelium-derived hyperpolarizing factor: where are we now? Arterioscler Thromb Vasc Biol 26 (6), 1215-25.

21. Hartmannsgruber, V. et al. (2007) Arterial response to shear stress critically depends on endothelial TRPV4 expression. PLoS One 2 (9), e827.

22. Sonkusare, S.K. et al. (2012) Elementary Ca2+ signals through endothelial TRPV4 channels regulate vascular function. Science 336 (6081), 597-601.

23. $\mathrm{Ma}$, X. et al. (2011) Heteromeric TRPV4-C1 channels contribute to store-operated $\mathrm{Ca}(2+)$ entry in vascular endothelial cells. Cell Calcium 50 (6), 502-9.

24. Ozkor, M.A. and Quyyumi, A.A. (2011) Endothelium-derived hyperpolarizing factor and vascular function. Cardiol Res Pract 2011, 156146.

25. Griffith, T.M. (2004) Endothelium-dependent smooth muscle hyperpolarization: do gap junctions provide a unifying hypothesis? Br J Pharmacol 141 (6), 881-903.

26. Shimokawa, H. (2014) 2014 Williams Harvey Lecture: importance of coronary vasomotion abnormalities-from bench to bedside. Eur Heart J 35 (45), 3180-93.

27. Sandow, S.L. and Hill, C.E. (2000) Incidence of myoendothelial gap junctions in the proximal and distal mesenteric arteries of the rat is suggestive of a role in endothelium-derived hyperpolarizing factor-mediated responses. Circ Res 86 (3), 341-6.

28. Brahler, S. et al. (2009) Genetic deficit of SK3 and IK1 channels disrupts the endothelium-derived hyperpolarizing factor vasodilator pathway and causes hypertension. Circulation 119 (17), 2323-32. 29. Chaston, D.J. et al. (2013) Polymorphism in endothelial connexin 40 enhances sensitivity to intraluminal pressure and increases arterial stiffness. Arterioscler Thromb Vasc Biol 33 (5), 962-70. 30. Goto, K. et al. (2018) Endothelium-Dependent Hyperpolarization (EDH) in Hypertension: The Role of Endothelial Ion Channels. Int J Mol Sci 19 (1).

31. Imig, J.D. (2016) Epoxyeicosatrienoic Acids and 20-Hydroxyeicosatetraenoic Acid on Endothelial and Vascular Function. Adv Pharmacol 77, 105-41.

32. Soler, A. et al. (2018) Elevated 20-HETE in metabolic syndrome regulates arterial stiffness and systolic hypertension via MMP12 activation. J Mol Cell Cardiol 117, 88-99.

33. Taddei, S. et al. (2006) Identification of a cytochrome P450 2C9-derived endothelium-derived hyperpolarizing factor in essential hypertensive patients. J Am Coll Cardiol 48 (3), 508-15. 34. Roca, F. et al. (2018) Evidence for a Role of Vascular Endothelium in the Control of Arterial Wall Viscosity in Humans. Hypertension 71 (1), 143-150.

35. Persy, V. and D'Haese, P. (2009) Vascular calcification and bone disease: the calcification paradox. Trends Mol Med 15 (9), 405-16.

36. Chen, J. et al. (2017) Coronary Artery Calcification and Risk of Cardiovascular Disease and Death Among Patients With Chronic Kidney Disease. JAMA Cardiol 2 (6), 635-643.

37. Guo, J. et al. (2017) Increased Aortic Calcification Is Associated With Arterial Stiffness Progression in Multiethnic Middle-Aged Men. Hypertension 69 (1), 102-108. 
38. Kamenskiy, A. et al. (2018) Prevalence of Calcification in Human Femoropopliteal Arteries and its Association with Demographics, Risk Factors, and Arterial Stiffness. Arterioscler Thromb Vasc Biol 38 (4), e48-e57.

39. Tsao, C.W. et al. (2014) Cross-sectional relations of arterial stiffness, pressure pulsatility, wave reflection, and arterial calcification. Arterioscler Thromb Vasc Biol 34 (11), 2495-500.

40. Durham, A.L. et al. (2018) Role of smooth muscle cells in vascular calcification: implications in atherosclerosis and arterial stiffness. Cardiovasc Res 114 (4), 590-600.

41. Alesutan, I. et al. (2016) Augmentation of phosphate-induced osteo-/chondrogenic transformation of vascular smooth muscle cells by homoarginine. Cardiovasc Res 110 (3), 408-18. 42. Kanno, Y. et al. (2008) Nitric oxide regulates vascular calcification by interfering with TGFsignalling. Cardiovasc Res 77 (1), 221-30.

43. Zhang, X. et al. (2016) Metformin alleviates vascular calcification induced by vitamin D3 plus nicotine in rats via the AMPK pathway. Vascul Pharmacol 81, 83-90.

44. Sambe, T. et al. (2018) Metformin treatment decreases nitroxidative stress, restores nitric oxide bioavailability and endothelial function beyond glucose control. Biomed Pharmacother 98, 149-156. 45. Zeisberg, E.M. et al. (2007) Discovery of endothelial to mesenchymal transition as a source for carcinoma-associated fibroblasts. Cancer Res 67 (21), 10123-8.

46. Man, S. et al. (2019) The therapeutic potential of targeting the endothelial-to-mesenchymal transition. Angiogenesis 22 (1), 3-13.

47. Medici, D. and Kalluri, R. (2012) Endothelial-mesenchymal transition and its contribution to the emergence of stem cell phenotype. Semin Cancer Biol 22 (5-6), 379-84.

48. Piera-Velazquez, S. and Jimenez, S.A. (2019) Endothelial to Mesenchymal Transition: Role in Physiology and in the Pathogenesis of Human Diseases. Physiol Rev 99 (2), 1281-1324.

49. Dejana, E. et al. (2017) The molecular basis of endothelial cell plasticity. Nat Commun 8, 14361. 50. Medici, D. et al. (2011) Transforming growth factor-beta2 promotes Snail-mediated endothelialmesenchymal transition through convergence of Smad-dependent and Smad-independent signalling. Biochem J 437 (3), 515-20.

51. Mani, S.A. et al. (2008) The epithelial-mesenchymal transition generates cells with properties of stem cells. Cell 133 (4), 704-15.

52. Zeisberg, E.M. et al. (2007) Endothelial-to-mesenchymal transition contributes to cardiac fibrosis. Nat Med 13 (8), 952-61.

53. Illigens, B.M. et al. (2017) Vascular Endothelial Growth Factor Prevents Endothelial-toMesenchymal Transition in Hypertrophy. Ann Thorac Surg 104 (3), 932-939.

54. Correia, A.C. et al. (2016) FGF2 inhibits endothelial-mesenchymal transition through microRNA20a-mediated repression of canonical TGF-beta signaling. J Cell Sci 129 (3), 569-79.

55. Borland, S.J. et al. (2017) Regulation of vascular smooth muscle cell calcification by syndecan4/FGF-2/PKCalpha signalling and cross-talk with TGFbeta. Cardiovasc Res 113 (13), 1639-1652.

56. Noseda, M. et al. (2004) Notch activation results in phenotypic and functional changes consistent with endothelial-to-mesenchymal transformation. Circ Res 94 (7), 910-7.

57. Hurlstone, A.F. et al. (2003) The Wnt/beta-catenin pathway regulates cardiac valve formation. Nature 425 (6958), 633-7.

58. Shore, E.M. et al. (2007) Corrigendum: A recurrent mutation in the BMP type I receptor ACVR1 causes inherited and sporadic fibrodysplasia ossificans progressiva. Nature Genetics 39, 276.

59. Medici, D. and Olsen, B.R. (2012) The role of endothelial-mesenchymal transition in heterotopic ossification. J Bone Miner Res 27 (8), 1619-22.

60. Yao, Y. et al. (2013) A role for the endothelium in vascular calcification. Circ Res 113 (5), 495-504. 61. Bostrom, K.I. et al. (2011) Activation of vascular bone morphogenetic protein signaling in diabetes mellitus. Circ Res 108 (4), 446-57.

62. Yao, J. et al. (2015) Serine Protease Activation Essential for Endothelial-Mesenchymal Transition in Vascular Calcification. Circ Res 117 (9), 758-69. 
63. Wermuth, P.J. et al. (2016) Stimulation of Transforming Growth Factor-beta1-Induced Endothelial-To-Mesenchymal Transition and Tissue Fibrosis by Endothelin-1 (ET-1): A Novel Profibrotic Effect of ET-1. PLoS One 11 (9), e0161988.

64. O'Riordan, E. et al. (2007) Chronic NOS inhibition actuates endothelial-mesenchymal transformation. Am J Physiol Heart Circ Physiol 292 (1), H285-94.

65. Sharma, S. et al. (2019) TRPV4 regulates matrix stiffness and TGFbeta1-induced epithelialmesenchymal transition. J Cell Mol Med 23 (2), 761-774.

66. Mahler, G.J. et al. (2014) Effects of shear stress pattern and magnitude on mesenchymal transformation and invasion of aortic valve endothelial cells. Biotechnol Bioeng 111 (11), 2326-37. 67. Balachandran, K. et al. (2011) Cyclic strain induces dual-mode endothelial-mesenchymal transformation of the cardiac valve. Proc Natl Acad Sci U S A 108 (50), 19943-8. 68. Zhong, A. et al. (2018) The Roles of Matrix Stiffness and ss-Catenin Signaling in Endothelial-toMesenchymal Transition of Aortic Valve Endothelial Cells. Cardiovasc Eng Technol 9 (2), 158-167. 69. Opdebeeck, B. et al. (2019) Indoxyl Sulfate and p-Cresyl Sulfate Promote Vascular Calcification and Associate with Glucose Intolerance. J Am Soc Nephrol 30 (5), 751-766.

70. Iyemere, V.P. et al. (2006) Vascular smooth muscle cell phenotypic plasticity and the regulation of vascular calcification. J Intern Med 260 (3), 192-210.

71. Muller, K.H. et al. (2019) Poly(ADP-Ribose) Links the DNA Damage Response and Biomineralization. Cell Rep 27 (11), 3124-3138.e13.

72. Chen, N.X. et al. (2018) Matrix vesicles induce calcification of recipient vascular smooth muscle cells through multiple signaling pathways. Kidney Int 93 (2), 343-354.

73. Simionescu, A. et al. (2005) Elastin-derived peptides and TGF-beta1 induce osteogenic responses in smooth muscle cells. Biochem Biophys Res Commun 334 (2), 524-32.

74. Hecht, E. et al. (2016) The matrix metalloproteinases 2 and 9 initiate uraemic vascular calcifications. Nephrol Dial Transplant 31 (5), 789-97.

75. Meng, F. et al. (2018) Endothelial Cells Promote Calcification in Aortic Smooth Muscle Cells from Spontaneously Hypertensive Rats. Cell Physiol Biochem 49 (6), 2371-2381.

76. Xie, S.A. et al. (2018) Matrix stiffness determines the phenotype of vascular smooth muscle cell in vitro and in vivo: Role of DNA methyltransferase 1. Biomaterials 155, 203-216.

77. Raaz, U. et al. (2015) Transcription Factor Runx2 Promotes Aortic Fibrosis and Stiffness in Type 2 Diabetes Mellitus. Circ Res 117 (6), 513-24.

78. Wang, Y. et al. (2018) Arterial Wall Stress Induces Phenotypic Switching of Arterial Smooth Muscle Cells in Vascular Remodeling by Activating the YAP/TAZ Signaling Pathway. Cell Physiol Biochem 51 (2), 842-853.

79. Kim, N.G. and Gumbiner, B.M. (2015) Adhesion to fibronectin regulates Hippo signaling via the FAK-Src-PI3K pathway. J Cell Biol 210 (3), 503-15.

80. Dupont, S. et al. (2011) Role of YAP/TAZ in mechanotransduction. Nature 474 (7350), 179-83. 81. Elosegui-Artola, A. et al. (2017) Force Triggers YAP Nuclear Entry by Regulating Transport across Nuclear Pores. Cell 171 (6), 1397-1410.e14.

82. Pan, J.X. et al. (2018) YAP promotes osteogenesis and suppresses adipogenic differentiation by regulating beta-catenin signaling. Bone Res 6,18 .

83. Tang, Y. et al. (2016) Snail/Slug binding interactions with YAP/TAZ control skeletal stem cell selfrenewal and differentiation. Nat Cell Biol 18 (9), 917-29.

84. G, C. et al. (2006) Disruption of clc-5 leads to a redistribution of annexin A2 and promotes calcium crystal agglomeration in collecting duct epithelial cells. Cell. Mol. Life. Sci 63, 367-377.

85. Karki, P. and Birukova, A.A. (2018) Substrate stiffness-dependent exacerbation of endothelial permeability and inflammation: mechanisms and potential implications in ALI and PH (2017 Grover Conference Series). Pulm Circ 8 (2), 2045894018773044.

86. Peng, X. et al. (2003) Wall stiffness suppresses Akt/eNOS and cytoprotection in pulse-perfused endothelium. Hypertension 41 (2), 378-81.

87. Fulton, D. et al. (1999) Regulation of endothelium-derived nitric oxide production by the protein kinase Akt. Nature 399 (6736), 597-601. 
88. Scotland, R.S. et al. (2002) Functional reconstitution of endothelial nitric oxide synthase reveals the importance of serine 1179 in endothelium-dependent vasomotion. Circ Res 90 (8), 904-10. 89. Chaterji, S. et al. (2014) Synergistic effects of matrix nanotopography and stiffness on vascular smooth muscle cell function. Tissue Eng Part A 20 (15-16), 2115-26.

90. Oh, R.S. et al. (2018) RNAi screening identifies a mechanosensitive ROCK-JAK2-STAT3 network central to myofibroblast activation. J Cell Sci 131 (10).

91. Zhang, Q. et al. (2018) Neural Crest Stem-Like Cells Non-genetically Induced from Human GingivaDerived Mesenchymal Stem Cells Promote Facial Nerve Regeneration in Rats. Mol Neurobiol 55 (8), 6965-6983.

92. Wang, C. et al. (2019) Poly(ADP-ribose) polymerase 1 accelerates vascular calcification by upregulating Runx2. Nat Commun 10 (1), 1203.

93. Yokoyama, M. and Hirata, K. (2007) Endothelial nitric oxide synthase uncoupling: Is it a physiological mechanism of endothelium-dependent relaxation in cerebral artery? Cardiovasc Res 73 (1), 8-9.

94. Ding, Y. et al. (2019) Peroxynitrite-Mediated SIRT (Sirtuin)-1 Inactivation Contributes to NicotineInduced Arterial Stiffness in Mice. Arterioscler Thromb Vasc Biol, Atvbaha118312346.

95. Tsihlis, N.D. et al. (2011) Nitric oxide inhibits vascular smooth muscle cell proliferation and neointimal hyperplasia by increasing the ubiquitination and degradation of $\mathrm{UbcH} 10$. Cell Biochem Biophys 60 (1-2), 89-97.

96. De Mey, J.G. and Vanhoutte, P.M. (2014) End o' the line revisited: moving on from nitric oxide to CGRP. Life Sci 118 (2), 120-8. 


\section{Glossary}

607

608

609

610

611

612

613

614

615

616

617

618

619

620

621

622

623

624

625

626

627

Cardiac cushion: a subset of cells that is found in embryonic heart tissue and plays a pivotal role in the development of the endocardium.

Carotid-femoral pulse wave velocity: a measure of 'central' arterial stiffness which is determined by measuring the transit time of the pulse wave and distance between the carotid and femoral artery. It is currently the gold standard with the best predictability for cardiovascular outcome.

Coupled state: refers to sufficient cofactors (e.g. tetrahydrobiopterin) and substrate (L-Arginine) being present to facilitate production of the vasoactive molecule nitric oxide (NO) by NO synthase (NOS).

Cyclic stress: repetitive occurrence of forces acting on the arterial vessel wall as a result of the pulsatile cardiac output.

Cyclic stretch: repetitive stretching of the arterial wall as a result of pulsatile cardiac output.

Cytochrome P450 (CYP450): a family of enzymes which function as monooxygenases. In the context of this review they are important in the production of bioactive arachidonic acid metabolites.

Endothelial to mesenchymal transition (EndMT): phenotypic switch of an endothelial cell to a mesenchymal stem-like cell.

Fibrodysplasia ossificans progressiva (FOP): patients suffering from ectopic ossification, caused by an autosomal dominant heterozygous germ-line mutation in the activin receptor-like kinase-2 (ALK2). 
628 Impedance: a measure of the resistance to flow in the arterial system.

629 Impedance mismatch: a mismatch which occurs at regions in the arterial tree where 630 wall properties and/or vessel diameter change.

Klotho deficient mice: a mouse strain that develops an accelerated ageing

632 phenotype with multiple ageing-related disorders, such as infertility, arterial media 633 calcification, skin atrophy and osteoporosis, caused by the absence of Klotho.

Matrix metalloproteinases (MMPs): a family of endopeptidases who degrade extracellular matrix proteins, especially collagen and elastin.

Metformin: a prescription drug that lowers blood glucose levels and is mainly used by type 2 diabetes patients.

Minocycline: a prescription drug, widely used as broad-spectrum antibiotic.

Myoendothelial gap junctions (MEGJ): membrane channels which provide hetero-

640

641

642

643

644 cellular coupling between endothelial (ECs) and vascular smooth muscle cells (VSMCs), allowing passage of small molecules.

$5 / 6^{\text {th }}$ nephrectomized mice: a mouse model that mimics the progressive nephron loss observed in chronic kidney disease patients. These mice undergo a subtotal $\left(2 / 3^{\text {th }}\right)$ removal of one kidney and total removal of the contralateral kidney. Administration of a high phosphorus diet to $5 / 6^{\text {th }}$ nephrectomised mice induces the development of arterial media calcification.

Obese Zucker rats: Zucker fatty rats have a mutated leptin receptor leading to a spontaneous development of obesity around 4 weeks of age. These rats suffer from hyperphagia, hyperinsulinemia and hyperlipidemia. 
650 Osteochondrogenic transdifferentiation: phenotypic switch of the vascular smooth

651 muscle cell to a cell with an osteoblastic or chondrocytic phenotype.

652 Pulse pressure: the difference between systolic and diastolic blood pressure.

653 Vessel tonus: the degree of basal constriction tone experienced by a blood vessel

654 relative to its maximally dilated state. Vasoactive molecules modulate vessel tone. 


\section{Box 1: The Arterial Stiffness Gradient}

656

657

658

659

660

661

662

663

664

665

666

667

668

669

670

671

672

673

674

675

676

677

Due to arterial system heterogeneity a progressive stiffness gradient is generated. As a result, the pulse wave velocity (PWV) increases progressively along the arterial tree [13]. These structural and functional distinguishable differences in the arterial tree have two important functions: (i) elastic arteries are important in converting the pulsatile pressure resulting from the ejection of blood during each cardiac cycle.

Stroke volume energy is stored in the wall of elastic arteries during systole.

Subsequently, elastic recoil of the aorta, during diastole, propels the blood on the basis of a pressure gradient, ensuring a continuous blood flow to the peripheral tissue and organs, (ii) muscular arteries are crucial players in blood pressure regulation. They are able to modulate the velocity of the incoming pressure wave by altering vessel tonus, i.e. contraction and relaxation of vascular smooth muscle cells (VSMCs). The pressure wave that is created during each cardiac cycle interacts with regions of impedance mismatch which arise due to varying properties of the vessel wall and vessel diameter. This in turn, results in partial wave reflections aimed towards the heart. Most wave reflections originate at the transition of low resistance (elastic) to high resistance (muscular) arteries or at branching origins of arterioles. Under physiological circumstances, the backward pressure wave (summations of wave reflections) arrives at the ascending aorta during diastole. These partial reflections are important to protect the microcirculation by limiting the transmission of pulsatile pressure energy to the periphery. The phase relationship of the forward and reflected wave determines the final amplitude and shape of the propagating pulse pressure. 
679 Classical mechanical forces (cyclic stress and shear stress) or vasoactive molecules 680 such as acetylcholine (ACh) and circulating hormones mediate vasodilation. The 681 predominant effect is an increase in activity of endothelial nitric oxide synthase 682 (eNOS). Important cofactors for NOS are tetrahydrobiopterin $\left(\mathrm{BH}_{4}\right), \mathrm{Ca}^{2+/}$ calmodulin 683 and flavin coenzymes. In its coupled state (in the presence of sufficient cofactors 684 and substrate), eNOS predominantly synthesizes nitric oxide (NO) from L-Arginine. $685 \mathrm{NO}$ and to a lesser extent, prostacyclin $\left(\mathrm{PGI}_{2}\right)$, are the main endothelium-derived 686 relaxing factors opposing the actions of endothelium-derived endothelin-1 (ET-1) and 687 (systemic) angiotensin II. NO diffuses into the sub-endothelial space and in VSMCs, 688 where it ultimately leads to cGMP-mediated relaxation of VSMCs [8]. Additionally, it 689 keeps the VSMC in a non-proliferative state [95]. Cyclooxygenase generated $\mathrm{PGI}_{2}$ 690 mediates VSMC relaxation through the generation of cAMP. ET-1, released by ECs, 691 primarily acts through its $\mathrm{ET}_{\mathrm{A}}$-receptor on VSMCs facilitating vasoconstriction. 692 Conversely, acting through ETB-receptors on ECs results in enhanced production of 693 NO. The balance between NO (relaxation) and ET-1 (contraction) is reviewed 694 elsewhere [96]. 


\section{Box 3: Clinician's Corner}

696

697

698

699

700

701

702

703

704

705

706

707

708

709

710

711

712

713

714

715

716

717

Arterial stiffness and arterial media calcification (AMC) are two important predictors of cardiovascular morbidity and mortality and initiate end-organ failure including severe brain and kidney damage. Until now, no effective treatments for both arterial stiffness and AMC are available.

Regulation of arterial compliance clearly relies on the vessel type and must be seen as a summation of the complex interaction between large elastic and smaller muscular arteries of the periphery. Therefore, peripheral measurements related to arterial stiffness (such as classical blood pressure measurements) must be interpreted with caution.

Arterial stiffness and AMC reinforce one another in which phenotypic alterations of ECs and VSMCs are essential. Anti-hypertensive drugs have beneficial effects by lowering arterial stiffness; however fail to preserve large artery distensibility. This implies that large intervention studies are needed in which not only systolic and diastolic blood pressure as well as PWV is measured but also noninvasive/invasive assessments of both endothelial function and VSMC tonus are determined.

Unraveling the molecular signatures and signaling pathways responsible for an increase in stiffness and AMC in large arteries will ultimately translate to better treatment options being available to patients at risk (i.e. chronic kidney disease and diabetes patients).

Potential upcoming strategies to prevent arterial stiffness and AMC include metformin (anti-diabetic drug) and minocycline (broad spectrum antibiotic). Both drugs are often used in the clinic and have proven to exert pleiotropic effects including the inhibition 
718 of $\mathrm{AMC}$ and arterial stiffness through respectively interfering with NO production and 719 phenotypic transition of VSMCs. 


\section{Figure legends}

721

722

723

724

725

726

727

728

729

730

731

732

733

Figure 1 - Key Figure

\section{Endothelial Cells And Vascular Smooth Muscle Cells Contribute to Arterial}

Stiffness and Arterial Media Calcification (AMC). (A) The vicious cycle of arterial stiffness and AMC with its stimulating events and clinical outcomes. (B) Matrix stiffness decreases the NO production leading to transdifferentiation of VSMCs into hypercontractile VSMCs (left) and ECs into mesenchymal stem cells (EndMT, right). In VSMCs, declined NO production activates the RhoA/ROCK pathway (orange) and TGF- $\beta$ signaling (blue). Their downstream effectors including YAP/TAZ, phosphorylated STAT3 and SMAD2/3 respectively upregulate osteochondrogenic marker genes (Runx2, BMP2 and Sox9) and thus the release of calcifying exosomes and Runx2-dependent fibrosis of the extracellular matrix (ECM). Matrix degradation by matrix metalloproteinase-2 and 9 (MMP-2/9, brown pacman shapes) enables migration of ECs and promote ECM mineralization. In ECs, decreased NO production is also associated with the RhoA/ROCK pathway resulting in activation of YAP/TAZ which interacts with EndMT inducers Snail, Slug and Twist as well as it might induce Runx2-dependent fibrosis and mineralization of the ECM. TGF- $\beta 1 / 2$ and BMP2/4 interact with activin-like kinase 2 and 5 (ALK2/5) activating SMAD1/5/8 and SMAD2/3 signaling to promote EndMT. Subsequently, the mesenchymal stem cell might transdifferentiate into osteochondrogenic cells (bottom center) and myofibroblasts (bottom right) favoring mineralization and fibrosis of the ECM, respectively. Three potential therapeutic targets (light green) are shown (i) metformin, altering NO and peroxynitrite (ONOO-) production, (ii) fibroblast growth factor-2 (FGF-2), inhibiting EndMT and VSMC mineralization and (iii) poly-ADP-ribose polymerase enzyme 
(PARP1) inhibitors, blocking STAT3-dependent ECM calcification. Dashed lines represent relevant findings in other cell-types.

\section{Figure 2. Important Players in Vessel Tone Regulation and Their Contribution to} Arterial Stiffening. The contribution of nitric oxide (NO), produced by endothelial NO synthase (eNOS) is larger in arteries proximal to the heart (left) while the endothelial dependent hyperpolarization (EDH) phenomenon regulates vasorelaxation predominantly in smaller, more distal arteries (right). Both mechanical stimuli (shear stress, cyclic stress) and vasoactive molecules such as acetylcholine (ACh) influence vessel tone. Vascular smooth muscle cell (VSMC) relaxation is mediated by hyperpolarization/reduction in intracellular calcium. Vasoconstriction and increased vessel tone by ET-1 action on VSMC ETA receptors is accompanied by increased intracellular calcium concentration. ET-1 binding on endothelial ETB receptors stimulates NO production by eNOS. EDH is initiated by an increase in endothelial intracellular calcium, crucial to facilitate potassium efflux from ECs (through calciumdependent potassium-channels - IK and SK). In turn, the latter induces potassium influx in VSMCs using three different channels/ATPase's. Hyperpolarization can also spread towards adjacent VSMCs via myoendothelial gap junctions (yellow flashes represent dispersion of the hyperpolarizing current). Red squares indicate modes of action by which arterial stiffness affects vessel tone regulation. A disturbed CYP450 metabolism (EETS/20-HETE) besides impaired NO bioavailability and EDH efficiency promotes a 'pro stiffening' phenotype. The inhibitory function of NO on ET-1 is diminished resulting in enhanced $\mathrm{ET}_{\mathrm{A}}$ receptor activity.

\section{Figure 3. Schematic Overview of Vascular Endothelial to Mesenchymal} Transition (EndMT). Various stimuli are able to induce EndMT. Best described is transforming growth factor beta (TGF- $\beta$ ) and bone morphogenetic protein (BMP) 
mediated EndMT through SMAD signaling and subsequent activation of transcription factors Snail, Slug and Twist. Wnt/ $\beta$-catenin and notch signaling may also play a role in EndMT (dashed line). Endothelial cells (ECs) obtain an elongated phenotype losing their cell-cell connections. Matrix degradation by matrix metalloproteinase-2 (MMP-2) and MMP-9 (brown pacman shapes) enables migration of ECs. The 'prostiffening/calcification' EC phenotype, i.e. reduction of nitric oxide (NO) bioavailability and increased endothelin-1 (ET-1) production promotes mesenchymal transition by inducing TGF- $\beta$ signaling. In a similar fashion, reduction of mineralization inhibitor matrix gla protein (MGP) results in enhanced BMP2 signaling. Inhibitors of EndMT include fibroblast growth factor-2 (FGF-2), vascular endothelial growth factor (VEGF) and BMP7. Mesenchymal stem-like cells can further differentiate into vascular smooth muscle cells (VSMCs), (myo)fibroblasts and osteoblasts.

\section{Figure 4 The Vicious Cycle Between Arterial Stiffness and Arterial Media}

Calcification. Under certain risk factors (i.e. high phosphate and calcium levels, inflammation, oxidative stress) vascular smooth muscle cells (VSMCs) transdifferentiate into osteochondrogenic cells with upregulation of Runx2, BMP2 and Sox9 mRNA expression (lower left). These VSMCs produce calcifying exosomes, initiating a cascade effect by stimulating neighboring VSMCs to release intracellular endoplasmic reticulum calcium stores through activation of the NAPDH oxidase and MAPK pathways, and calcifying exosomes. In the end the extracellular matrix (ECM) becomes mineralized and thus ECM stiffness occurs. Secondly, ECM stiffness influences in- and directly VSMC behavior. Matrix stiffness also stimulates the activation of the integrin-FAK-Src pathway leading to LATS1/2 inhibition and favoring the activation/translocation to the nucleus of YAP/TAZ proteins with potentially inducing Runx2 and $\beta$-catenin expression (right). The cell nucleus flattens and 
stretches its pores as a response to ECM stiffness triggering more influx of YAP/TAZ

795 and maybe other molecules which could alter VSMC phenotype. Indirectly, endothelial cells produce less NO during ECM stiffness, stirring the VSMC into a hypercontractile phenotype. This phenotypic state triggers the RhoA/ROCK pathway 798 which might alter either YAP activation and phosphorylation of STAT3. This latter 799 protein translocates into the nucleus to stimulate Runx2 expression. Finally, the 800 transcription factor Runx2 induces fibrosis/stiffness and mineralization of the ECM.

801 Matrix stiffness also directly triggers downregulation of DNA methyltransferase 1 802 (DNMT1) in VSMCs resulting in epigenetic DNA alterations and expression of bone803 like marker genes Runx2 and BMP2 (lower right). 